\title{
An Evolutionary Approach to Market Adoption of I-o-T
}

\author{
Frank T. Lorne* \\ School of Management, New York Institute of Technology-Vancouver \\ Vancouver, B.C. V7Y 1K8, Canada \\ e-mail: florne@nyit.edu
}

\begin{abstract}
An evolutionary approach suggested in Alchian (1950) will be used to provide a new framework for understanding the emergence of the ecosystem of I-o-T in the construction industry. The framework entails a measure of buyers and sellers complementarity and the focus of how information in the industry are shared. The concept of Smart Construction Objects (SCO) is found useful in delineating the role of information in shaping the emergence of an ecosystem via the interplay of the optimum distributions of players.
\end{abstract}

Keywords-Optimum distribution; Innovation ecosystem; Construction industry; Internet of things

\section{INTRODUCTION}

Considerable interests on the hardware and software development in the area of internet of things (I-o-T) have evolved in recent years, spanning a large variety of applications and involving numerous industries, (Harvard Business Review, 2014). The mechanism addressing to the entry point of applications have seldom been studied. This paper utilizes some recent concepts in classifying innovation ecosystems in addressing to how smart construction objects (SCOs) might have evolved or will be evolving in the construction industries. I-o-T may be a small part of the construction industry presently, but companies striving to compete to achieve a competitive advantage have to attend to these technological changes, many of which aim to reduce energy consumption and environmental concerns.

\section{EVOLUTIONARY APPROACH IN ECONOMICS}

The evolutionary approach to market adoption of new technology has long been formulated as a stochastic process with assumed probability distributions. This approach has its origin in a classic paper by a UCLA economist, Armen Alchian. In a 1950 piece entitled "Uncertainty, Evolution, and Economic Theory", Alchian probed into economists' neoclassical assumption of profit maximization as a behavior postulate for firms and individuals. He specifically pointed out that "profit maximization is meaningless as a guide to specifiable action" (p. 211). The essence of decision making under uncertainty is the "adaptive, imitative, and trial-and error behavior in the pursuit of positive profits". It is widely believed that the essence of this evolutionary approach has been embedded and well formulated by many writers in evolutionary economics in terms of various stochastic specifications. A leading example of this can be found in Nelson and Winter, 1986.
Yet, arguably, Alchian had in mind a process quite different from those that had been formulated. He was explicit in this explanation in the 1950 article. Citing earlier work by G. Tintner's critical comments on profit maximization, and defining uncertainty as the consequence that the distribution of potential outcomes from decision making is overlapping, the more sensible behavior postulate being that actions (or decisions) are made for a potential outcome distribution that would be preferable. The word "preferable" is highlighted in this paper over a more stringent criterion of knowing what the maximum is. In Alchian's words,

"Essentially, the task is converted into making a decision (selecting an action) whose potential outcome distribution is preferable, that is, choosing the action with the optimum distribution, since there is no such thing as a maximizing distribution." P. 212.

Alchian's paper has been widely cited, but scholars working in the field of evolutionary economics have seldom explicitly addressed to his proposed behavior postulates. What have been widely adopted is to identify evolutionary economic outcomes as a stochastic process, e.g. a search characterized by a Markov process, and a selection characterized by price in the market, (see Nelson and Winter, p. 144-153). It is the opinion of this author that this mathematical formulation does not adequately captures the gist of Alchian's proposition.

\section{SCOS AND THE INNOVATION ECO-SYSTEMS}

This paper probes into the question of optimum distribution in the context of how smart construction objects, SCOs, are likely to be adopted in the construction industry. Useful characterization of information needed for effective use of I-o-T has been addressed recently in articles by Niu, et. al. $(2015,2016)$. Specifically, SCOs can be ranked in terms of its information processing capabilities in dimensions of awareness, communicativeness, and autonomy, for creating smart devices for sensing, processing, and also to interact with information with the environment where the devices are located all for improving decision makings.

It will be helpful to interpret the role of information generally in the topology of the innovation ecosystem of the construction industry as described in Soh and Wang (2016). Unlike conventional evolutionary models, the adoption of a particular I-o-T cannot be considered in isolation and on a piecemeal basis, as the adoption of a particular mechanism (or device) often depends on a variety of component 
suppliers and complementary products whose technology development and adoption are on-going processes also.

The players involved in such processes simultaneously create, diffuse, compete, and capture values. While disruptive technologies that change lifestyle in some fundamental way often happen, a majority of innovations could piggy back ride on existing technologies, emphasizing refinements and the achievement of simplicity. The selection of a platform, and how competition and cooperation can lead to the adoption of a popular platform is of utmost importance. This is the essence of the problem that Soh and Wang intended to study and to categorize, in terms of how information production is characterized, not so much in terms of the outcome of information and how they are embedded into physical processes and products.

The notion of optimum distribution comes into the above frameworks in some pragmatic ways that a framework describing an information topology can be studied. A player, be it a buyer or a producer, often does not know what is wanted or what needs to be produced. Either party is driven by some subjective notion of distribution of counterparties and possible partners. In that respect, the typology of innovation ecosystem characterized by Soh and Wang (2016) can be instructive in noting that the optimum distribution subjectively determined by a player can determine its prior position in a topology of the ecosystem. Whether a platform can indeed gather enough momentum (participation and subscription) will depend on how these optimum distributions evolves and whether counterparty interests overlap or not. The resultant optimum distribution will define the characteristics of the innovation ecosystem of I-o$\mathrm{T}$ in an industry.

A modification of Figure 1 in Soh and Wang serves to illustrate:

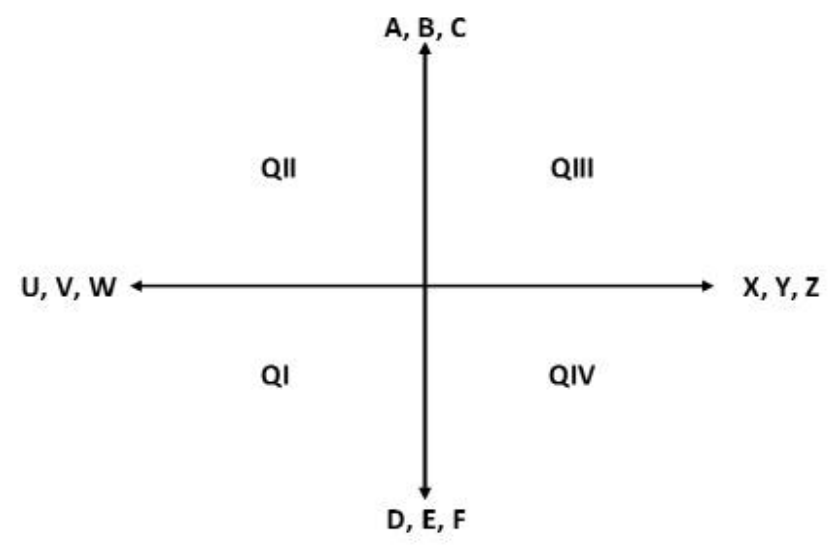

Figure 1.

On the space ranked by complementarity in the vertical dimension and knowledge configuration in the horizontal dimension, distribution of initial players can be assumed to be $\mathrm{A}$ to $\mathrm{F}$ in the vertical dimension, and from $\mathrm{U}$ to $\mathrm{Z}$ in the horizontal dimension. Each letter represents a player. Each player has its own optimum distribution, formulated based of his/her perceived probability of success of other players in the whole ecosystem. For example, let's say players U, V, W, all see great potentials with each other, but that the distribution of optimum distribution is completely diffused with $\mathrm{X}, \mathrm{Y}, \mathrm{Z}$; and players $\mathrm{D}, \mathrm{E}, \mathrm{F}$, all see the need of adopting a particular device to be so overwhelmingly strong in relationship to a completely diffused optimum distribution of A, B, C, it is likely that the ecosystem of that industry will be in the strong direction of quarter I (QI), which is a single product ecosystem.

Once the above example is understood, it is a small extension to see that most ecosystem would not fall into such extreme as described above, but as a push- and-pull game between the optimum distributions of players in the topology of the ecosystem. The next important feature that needs to be addressed to in the dynamics and the evolution of the industry optimum distribution is how information is being perceived, analyzed, communicated, and disseminated. This is the essence of the SCO capability demanded and supplied by each of the players in this topology. Niu, et. al. (2015, 2016) ranked I-o-T in terms of awareness, communicativeness, and autonomy, with information attributes categorized in some systematic and useful ways. The ultimate deciders in the usefulness of each attribute, and thus the adoption plane chosen for each I-o-T, are likely to be the buyers, i.e. players D, E, F in Figure 1. For example, for the logistics and supply chain management (LSCM) construction I-o-Ts, Niu, et. al. observed that it is the activity awareness-pull communicativeness-active autonomy plane that the winning I-o-T is most likely to evolve. This observation came from the push and pull of optimum distribution of the players in Figure 1.

\section{CONCLUSION}

It is the main conclusion and the highlight of this paper that Alchian's concept of optimum distribution should be studied for a better understanding of how the ecosystem I-o$\mathrm{T}$ in a particular industry would evolve. The construction industry is probably the most futile ground to study how I-o$\mathrm{T}$ are being adopted. This paper provides an integrative framework to further study specific examples that can be at a primitive stage of experimentation as well as sophisticated technologies that have been widely adopted

\section{ACKNOWLEDGMENT}

The author wishes to thank Remi Charron and Wilson Lu for discussion about the subject matter of this paper. Ling Ding graphical work, and traveling funding from the New York Institute of Technology, Global Program, is gratefully acknowledged.

\section{REFERENCES}

[1] Alchian, Armen. 1950. "Uncertainty, Evolution, and Economic Theory.” Journal of Political Economy. Vol. 58, No. 3 (Jun., 1950), pp. 211-221

[2] Soh, Pek Hooi and Tao Wang. 2016. "A Typology of InnovationEcosystems" (Competitive Advantage in Innovation Ecosystems) Conference Presentation at the Sixth Beedie Innovation Conference, CPA Innovation Center, Simon Fraser University, Sept. 29, 2016. 
[3] Niu, Yuhan; Weisheng Lu; Diandian Liu; Ke Chen; Chimay Anumba; and George G. Huang. 2016. "An SCO-Enabled Logistics and Supply Chain-Management System in Construction." Journal of Construction Engineering and Management, forthcoming

[4] Nelson, Richard R. and Winter, Sidney G. (1982). An Evolutionary Theory of Economic Change. Harvard University Press.
[5] Niu, Yuhan; Weisheng Lu; Ke Chen; George G. Huang; and Chimay Anumba. 2015. "Smart Construction Objects". Journal of Computing in Civil Engineering

[6] INTERNET OF THINGS: SCIENCE FICTION OR BUSINESS FACT? Harvard Business Review, 2014. 